\title{
Estrogen receptor 1 gene polymorphisms and coronary artery disease in the Brazilian population
}

S. Almeida and M.H. Hutz
Departamento de Genética, Instituto de Biociências,

Universidade Federal do Rio Grande do Sul, Porto Alegre, RS, Brasil

\section{Correspondence}

M.H. Hutz

Departamento de Genética

Instituto de Biociências, UFRGS

Caixa Postal 15053

91501-970 Porto Alegre, RS

Brasil

Fax: +55-51-3343-5850

E-mail: mara.hutz@ufrgs.br

Research supported by CNPq and PRONEX.

Received April 8, 2005

Accepted November 10, 2005

\begin{abstract}
We examined the association of three established single nucleotide polymorphisms, IVSI-397T >C, IVSI-35IA $>G$, and $+261 G>C$, in the ESR1 gene with the prevalence and severity of coronary atherosclerosis in a southern Brazilian population of European ancestry. Three hundred and forty-one subjects (127 women and 214 men) with coronary artery disease (CAD) were classified as having significant disease (CAD+ patient group) when they showed $60 \%$ or more luminal stenosis in at least one coronary artery or major branch segment at angiography; patients with $10 \%$ or less luminal stenosis were considered to have minimal CAD (CAD- patient group). The control sample consisted of 142 subjects (79 women and 63 men) without significant disease, in whom coronary angiography to rule out the presence of asymptomatic CAD was not performed. The polymorphisms were investigated by polymerase chain reaction followed by restriction analyses. In the male sample, the $+261 G>C^{*} C$ allele was more frequent in $\mathrm{CAD}+$ than $\mathrm{CAD}$ - subjects ( 8 versus $1 \%, \mathrm{P}=0.024$ ). Homozygosity for the C allele of the IVSI-397T $>C$ polymorphism was also significantly associated with increased CAD severity (OR: $2.99 ; 95 \% \mathrm{CI}=1.35-6.63 ; \mathrm{P}=0.007)$. In agreement with previous findings, these results suggest that the IVS1-397T>C${ }^{*} C$ allele was associated with $\mathrm{CAD}$ severity independent of gender, whereas the association of the $+261 G>C$ variant with CAD was observed in males only. The relation between ESR1 variation and CAD may influence clinical decisions such as the use of hormone therapy, and additionally will be helpful to identify the genetic susceptibility determinants of cardiovascular disease development.
\end{abstract}

\section{Introduction}

The cardioprotective effects of estrogen are known and may be mediated by systemic effects on lipid profile (1), coagulation and/ or fibrinolysis (2). However, the direct actions of estrogen on blood vessels might
Key words

- ESR1 polymorphisms

- Coronary artery disease

- Estrogen

- Hormonal status

........ contribute considerably to the cardioprotective effects of this hormone; estrogen increases vasodilatation, accelerates endothelial cell growth, and inhibits the migration and proliferation of smooth muscle cells in vitro and the response of blood vessels to injury and development of atherosclerosis 
(2). Estrogen may also be considered to have an anti-inflammatory action by decreasing several inflammation markers. Nevertheless, some studies have demonstrated a rise in C-reactive protein levels after initiation of oral estrogen plus progesterone replacement therapy in postmenopausal women $(3,4)$.

Ohlsson et al. (5) and Hodgin et al. (6) reported that the cholesterol-lowering effect of 17ß-estradiol in mice depends on the presence of estrogen receptor 1 (ESR1). Chen et al. (7) demonstrated that this receptor mediates the nongenomic activation of endothelial nitric oxide synthase.

Three polymorphisms have been extensively studied in the ESR1 gene, two in the first intron IVS1-397T >C (rs2234693) and IVS1-351A>G (rs9340799), and a silent mutation in exon $1,+261 G>C$ (rs746432). These variants were initially associated with onset of menopause (8), bone density $(9,10)$, hypertension (11), body mass index (12), cardiovascular disease, and intermediate cardiovascular markers (13-22). More recently a TA dinucleotide repeat polymorphism has been described and reported to be associated with the severity of cardiovascular disease $(23,24)$. Although several studies on association with cardiovascular disease have been performed, inconsistent results have been obtained. How and to what extent polymorphisms in the ESR1 gene might function as genetic markers of vascular pathology is also not known. Therefore, repeating the studies of the association of ESR1 polymorphisms with cardiovascular disease in diverse settings and populations is very important to assess if this genetic variability can influence the susceptibility to cardiovascular disease.

In the present study, we examined the association of three established single nucleotide polymorphisms (SNPs), IVS1-397T $>C$, $I V S 1-351 A>G$, and $+261 G>C$ in the ESR 1 gene with the prevalence and severity of coronary atherosclerosis in a southern Brazilian sample of European ancestry.

\section{Subjects and Methods}

\section{Patients and controls}

Coronaryartery disease sample. The study was conducted on 341 individuals (127 women and 214 men) of European descent, ascertained by morphological characteristics as previously described (25), who required coronary angiography due to symptoms related to coronary artery disease (CAD). These patients were of unrestricted age and were contacted at the Hospital de Clínicas de Porto Alegre (HCPA). Informed consent to provide a blood sample for DNA extraction to be used in studies approved by the Hospital Ethics Committee was obtained from each subject included in the sample. A questionnaire which included details on lifestyle variables such as smoking, physical activity, and anthropometric measures was completed by the subjects. Subjects were classified for smoking status as never smokers, and smokers (past or current smoking). Patients were classified as having significant disease (CAD+ patient group) if they showed $60 \%$ or more luminal stenosis in at least one coronary artery or major branch segment at angiography. Patients with $10 \%$ or less luminal stenosis were considered to have minimal CAD (CAD- patient group) and patients with an occlusion between 11 and 59\% were considered to have intermediate $\mathrm{CAD}$ and were not included in the severity analyses.

Control sample. The control sample consisted of 142 subjects ( 79 women and 63 men) of European descent. As done for the patients, the ethnicity of these individuals was classified in terms of physical characteristics. The subjects included in this sample were randomly selected from two clinical centers at the Federal University of Rio Grande do Sul among those who came from several city health centers for free routine blood determinations. Only those without significant disease were included. In these latter subjects, it was unethical to perform coronary angiography to rule out the presence of asymptomatic CAD. A question- 
naire which included details on drug intake and lifestyle variables such as smoking, physical activity, alcohol consumption, oral contraceptive use, menopause status, and anthropometric measures was completed. For smoking status, subjects were classified as never smokers and current smokers (ex-smokers were excluded). All individuals gave written informed consent prior to the investigation. Exclusion criteria were pregnancy, secondary hyperlipidemia due to renal, hepatic or thyroid disease, and diabetes or fasting blood glucose levels higher than $6.9 \mathrm{mmol} / \mathrm{L}$.

All subjects were studied in the morning after a 12-h fast. Weight was measured in subjects without shoes and wearing light clothing. Body height was determined without shoes, heels together, with the back to the wall. Body mass index was calculated as weight $/$ height $^{2}\left(\mathrm{~kg} / \mathrm{m}^{2}\right)$. Waist circumference was measured at the smallest horizontal circumference between the 12th rib and the iliac crest.

\section{DNA analyses}

Genomic DNA was extracted from peripheral blood leukocytes by the method of Lahiri and Nurnberger Jr. (26). The ESR1 SNPs (IVSI-397T>C, IVS1-351A $>G$, and $+261 G>C$ ) were amplified by the polymerase chain reaction using the same conditions and oligonucleotide primers as previously described $(10,27,28)$. The amplification products were subsequently digested with restriction enzymes under conditions recommended by the manufacturer: PvuII (IVS1-397T>C), XbaI (IVS1$351 A>G)$, and $B s t \mathrm{UI}(+261 G>C)$. The genotypes were determined after agarose gel electrophoresis with ethidium bromide, using a 50-bp ladder to score the band sizes.

\section{Biochemical analyses}

Blood samples were collected from patients after a 12-h fast. Total cholesterol, HDL-cholesterol, tryglyceride, and glucose levels were determined by standard methods using commercial kits. LDL-cholesterol was calculated according to the Friedewald formula (29). The lipid levels of patients and controls were analyzed by similar standard methods at two clinical centers of the Federal University of Rio Grande do Sul during the period from 2000 to 2001 on the same day of sample collection.

\section{Statistical analyses}

Allele frequencies at the individual loci were estimated by counting. The agreement of genotype frequencies with Hardy-Weinberg equilibrium expectations was tested using the chi-square test. The PEPI statistical program version 4.0 (30) was used to compare the frequencies among groups, adjusted residuals, and the power of the test. The groups were compared by the Student $t$ test or one-way analysis of variance using the Graph Pad InStat software version 2.04a (Graph Pad Software, San Diego, CA, USA). Multiple logistic regression was performed using the SPSS statistical package (SPSS ${ }^{\circledast}$ for Windows ${ }^{\mathrm{TM}}$, version 8.0). The maximum likelihood estimate of haplotype frequencies was calculated from multisite marker data using the Multiple Locus Haplotype Analysis software, version 2.0 (31-33). Linkage disequilibrium was tested by a $\chi^{2}$ goodness of fit test using the Arlequin program, version 2.000 (34). D' values, the relative magnitude of linkage disequilibrium, were calculated as described previously (35).

\section{Results}

The characteristics of patients and controls are reported in Table 1. CAD+ women were older than controls and CAD- women $(\mathrm{P}<0.001)$. The prevalence of hypertension was also higher in CAD+ women than in the other groups $(\mathrm{P}<0.001)$. CAD+ men were also older than controls and CAD- men $(\mathrm{P}=$ 0.019). Mean total cholesterol and LDL- 
Table 1. Characteristics of the participants of the present study.

\begin{tabular}{|c|c|c|c|c|c|c|c|c|}
\hline & \multicolumn{4}{|c|}{ Women } & \multicolumn{4}{|c|}{ Men } \\
\hline & Control & CAD- & $\mathrm{CAD}+$ & $P$ & Control & CAD- & CAD+ & $P$ \\
\hline Number & 80 & 69 & 60 & & 63 & 41 & 148 & \\
\hline Age (years) & $58 \pm 8.8^{a}$ & $58 \pm 10.6^{a}$ & $64 \pm 10.5^{b}$ & $<0.001$ & $59 \pm 11.2$ & $56 \pm 13.4$ & $61 \pm 10.4$ & 0.019 \\
\hline T-chol (mmol/L) & $5.88 \pm 1.39$ & $5.65 \pm 1.37$ & $6.01 \pm 1.27$ & 0.301 & $5.39 \pm 1.19 c, d$ & $4.98 \pm 1.04^{c}$ & $5.60 \pm 1.18^{d}$ & 0.011 \\
\hline HDL-C (mmol/L) & $1.16 \pm 0.31$ & $1.24 \pm 0.30$ & $1.19 \pm 0.26$ & 0.275 & $1.03 \pm 0.30$ & $1.03 \pm 0.29$ & $0.98 \pm 0.26$ & 0.354 \\
\hline LDL-C (mmol/L) & $3.84 \pm 1.23$ & $3.66 \pm 2.02$ & $3.94 \pm 1.07$ & 0.551 & $3.48 \pm 1.06^{e, f}$ & $3.22 \pm 0.84^{e}$ & $3.76 \pm 1.04^{f}$ & 0.006 \\
\hline $\mathrm{TG}(\mathrm{mmol} / \mathrm{L})$ & $1.94 \pm 1.30$ & $1.59 \pm 0.88$ & $1.98 \pm 1.08$ & 0.083 & $1.90 \pm 1.14$ & $1.77 \pm 1.40$ & $1.88 \pm 0.94$ & 0.805 \\
\hline BMI $\left(\mathrm{kg} / \mathrm{m}^{2}\right)$ & $27.3 \pm 4.2$ & $27.8 \pm 5.2$ & $27.0 \pm 4.5$ & 0.609 & $27.1 \pm 3.7$ & $26.7 \pm 3.7$ & $28.0 \pm 4.4$ & 0.122 \\
\hline Sedentary (\%) & 67 & 70 & 72 & 0.798 & 57 & 52 & 61 & 0.575 \\
\hline Smokers and ex-smokers (\%) & 21 & 32 & 37 & 0.104 & $34^{\mathrm{h}}$ & 58 & $74^{\mathrm{h}}$ & $<0.001$ \\
\hline Hypertension (\%) & $40^{\mathrm{h}}$ & 61 & $78^{\mathrm{h}}$ & $<0.001$ & $32^{h}$ & 53 & 599 & 0.002 \\
\hline Postmenopausal (\%) & - & 75 & 86 & - & - & - & - & - \\
\hline Diabetes (\%) & 15 & 9 & 22 & 0.111 & 22 & 9 & 17 & 0.254 \\
\hline
\end{tabular}

Unadjusted data and continuous data are reported as mean $\pm \mathrm{SD}$. T-chol = total cholesterol; HDL-C $=\mathrm{HDL}$-cholesterol; $\mathrm{LDL}-\mathrm{C}=\mathrm{LDL}-\mathrm{cholesterol}$; $\mathrm{TG}=$ triglycerides; $\mathrm{BMI}=$ body mass index. $\mathrm{CAD}-=$ minimal coronary artery disease; $\mathrm{CAD}+=$ coronary artery disease. Data were analyzed by one-way analysis of variance followed by the Tukey post hoc test when necessary for continuous variables and by the chi-square test followed by adjusted residual analysis for categorical values.

a-fTukey HSD test, $\mathrm{P}<0.01$. gAdjusted residual $\mathrm{P}<0.01$. hAdjusted residual $\mathrm{P}<0.001$.

Table 2. Allele and genotype frequencies of $+261 G>C, I V S 1-397 T>C$ and $I V S 1-351 A>G$ polymorphisms in the ESR 1 gene in the study groups separated by gender.

\begin{tabular}{|c|c|c|c|c|c|c|c|c|c|c|c|c|c|c|}
\hline & \multicolumn{7}{|c|}{ Women } & \multicolumn{7}{|c|}{ Men } \\
\hline & \multicolumn{2}{|c|}{ Control } & \multicolumn{2}{|c|}{ CAD- } & \multicolumn{2}{|c|}{$\mathrm{CAD}+$} & \multirow[t]{2}{*}{$P$} & \multicolumn{2}{|c|}{ Control } & \multicolumn{2}{|c|}{ CAD- } & \multicolumn{2}{|c|}{ CAD+ } & \multirow[t]{2}{*}{$\mathrm{P}$} \\
\hline & $F(\%)$ & $\mathrm{N}$ & $\mathrm{F}(\%)$ & $\mathrm{N}$ & $F(\%)$ & $\mathrm{N}$ & & $\mathrm{F}(\%)$ & $\mathrm{N}$ & $\mathrm{F}(\%)$ & $N$ & $F(\%)$ & $N$ & \\
\hline \multicolumn{15}{|l|}{$+261 G>C$} \\
\hline Number & \multicolumn{2}{|c|}{79} & \multicolumn{2}{|c|}{68} & \multicolumn{2}{|c|}{59} & & \multicolumn{2}{|c|}{63} & \multicolumn{2}{|c|}{43} & \multicolumn{2}{|c|}{148} & \\
\hline $\mathrm{C} / \mathrm{C}$ & 0.0 & 0 & 0.0 & 0 & 0.0 & 0 & & 0.0 & 0 & 0.0 & 0 & 1.0 & 1 & \\
\hline $\mathrm{C} / \mathrm{G}$ & 6.0 & 5 & 9.0 & 6 & 15.0 & 9 & 0.206 & 8.0 & 5 & 2.0 & $1^{\mathrm{a}}$ & 15.0 & $23^{a}$ & 0.025 \\
\hline $\mathrm{G} / \mathrm{G}$ & 94.0 & 74 & 91.0 & 62 & 85.0 & 50 & & 92.0 & 58 & 98.0 & $42^{a}$ & 84.0 & $124^{a}$ & \\
\hline $\mathrm{G}$ & 97.0 & 153 & 96.0 & 130 & 92.0 & 109 & 0.223 & 96.0 & 121 & 99.0 & $85^{a}$ & 92.0 & $271^{b}$ & 0.024 \\
\hline \multicolumn{15}{|c|}{$I V S 1-397 T>C$} \\
\hline Number & \multicolumn{2}{|c|}{80} & \multicolumn{2}{|c|}{69} & \multicolumn{2}{|c|}{60} & & \multicolumn{2}{|c|}{63} & \multicolumn{2}{|c|}{43} & \multicolumn{2}{|c|}{150} & \\
\hline$T / T$ & 35.0 & 28 & 36.0 & 25 & 27.0 & 16 & & 41.0 & 26 & 37.0 & 16 & 37.0 & 56 & \\
\hline $\mathrm{T} / \mathrm{C}$ & 51.0 & 41 & 51.0 & 35 & 48.0 & 29 & 0.129 & 49.0 & 31 & 58.0 & 25 & 45.0 & 67 & 0.071 \\
\hline $\mathrm{C} / \mathrm{C}$ & 14.0 & 11 & 13.0 & 9 & 25.0 & 15 & & 10.0 & 6 & 5.0 & 2 & 18.0 & 27 & \\
\hline $\mathrm{T}$ & 61.0 & 97 & 62.0 & 85 & 51.0 & 61 & 0.156 & 66.0 & 83 & 66.0 & 57 & 60.0 & 179 & 0.341 \\
\hline \multicolumn{15}{|c|}{ IVS1-351A>G } \\
\hline Number & \multicolumn{2}{|c|}{80} & \multicolumn{2}{|c|}{71} & \multicolumn{2}{|c|}{60} & & \multicolumn{2}{|c|}{63} & \multicolumn{2}{|c|}{43} & \multicolumn{2}{|c|}{150} & \\
\hline $\mathrm{G} / \mathrm{G}$ & 43.0 & 34 & 45.0 & 31 & 38.0 & 23 & & 52.0 & 33 & 42.0 & 18 & 49.0 & 74 & \\
\hline $\mathrm{G} / \mathrm{A}$ & 51.0 & 41 & 45.0 & 31 & 49.0 & 29 & 0.649 & 43.0 & 27 & 56.0 & 24 & 40.0 & 60 & 0.165 \\
\hline $\mathrm{A} / \mathrm{A}$ & 6.0 & 5 & 10.0 & 9 & 13.0 & 8 & & 5.0 & 3 & 2.0 & 1 & 11.0 & 16 & \\
\hline $\mathrm{G}$ & 68.0 & 109 & 67.0 & 93 & 62.0 & 75 & 0.581 & 74.0 & 93 & 70.0 & 60 & 69.0 & 208 & 0.643 \\
\hline
\end{tabular}


cholesterol levels were higher in CAD+ men than in CAD- men. The prevalence of smokers and ex-smokers, as well as hypertension, was higher in $\mathrm{CAD}+$ men.

Allele and genotype frequencies for the ESR1 variants in all subsamples are shown in Table 2 stratified by gender. The genotype frequencies observed for all polymorphisms studied did not differ significantly from those expected under Hardy-Weinberg equilibrium. Regarding the $+261 G>C$ polymorphism only one $\mathrm{C} / \mathrm{C}$ homozygote was detected in the samples studied; therefore, for statistical analysis purposes this subject was assigned to the G/C group. No statistically significant difference was detected in allele or genotype (Table 2) frequencies among the three female groups. But in the male sample, the $+261 G>C^{*} C$ allele was more frequent in $\mathrm{CAD}+$ than $\mathrm{CAD}$ - subjects $(8$ versus $1 \%, \mathrm{P}=0.024)$. The power of the test was $80 \%$.

The effects of the ESR1 genotypes on CAD severity were also evaluated using the percentage of luminal stenosis as the dependent variable (CAD- $\leq 10 \%$ and CAD+ $\geq 60 \%$ ) in a multiple logistic regression analysis. In the whole sample, the variables that remained statistically associated with CAD severity are shown in Table 3. After controlling for age, gender, hypercholesterolemia and smoking, homozygosity for the IVSI$397 T>C^{*} C$ allele was found to be significantly associated with increased CAD severity (OR: 2.99; 95\% CI = 1.35-6.63; $\mathrm{P}=0.007)$.

Seven ESR1 haplotypes were detected in the investigated sample (data not shown). The two most common haplotypes were $+261 G$ - IVS1-397T - IVS1-351A and +261G - IVS1-397C - IVS1-351G, and these two combinations accounted for 58 and $33 \%$ of the investigated chromosomes, respectively. Linkage disequilibrium was detected between pairwise combinations and D' values were: $+261 G>C-I V S 1-397 T>C, 0.51$ $\left(\chi_{\text {d.f. }=1}^{2}=17.25 ; \mathrm{P}<0.001\right),+261 G>C-$
IVS1-351A $>G, 0.82\left(\chi_{\text {d.f. }=1}^{2}=6.84 ; \mathrm{P}=\right.$ 0.009) and IVS1-397T $>C-I V S 1-351 A>G$, $0.98\left(\chi_{\text {d.f. }=1}^{2}=392.10 ; \mathrm{P}<0.001\right)$. When compared to all other haplotypes, haplotype +261G - IVS1-397T - IVS1-351A was less frequent in $\mathrm{CAD}+(0.568)$ than in $\mathrm{CAD}$ subjects (0.620) and controls $(0.630 ; \mathrm{P}=$ 0.034; Table 4). ESR1 genotypes and haplotypes were not associated with lipid levels in the present study (data not shown).

\section{Discussion}

Recently two large, well-designed and well-implemented studies were published with apparent contradictory results in rela-

Table 3. Multiple logistic regression model and prediction of variables for coronary atherosclerosis disease severity using the percentage of luminal stenosis as the dependent variable [CAD- $\leq 10 \%(N=112)$ and $C A D+\geq 60 \%(N=210)]$.

\begin{tabular}{llll}
\hline $\begin{array}{l}\text { IVS1-397T }>C \text { model } \\
\text { Variable }\end{array}$ & OR & $95 \% \mathrm{Cl}$ & $\mathrm{P}$ \\
\hline Age $(>60$ years) & 2.65 & $1.6-4.5$ & $<0.001$ \\
Gender (male) & 4.38 & $2.5-7.7$ & $<0.001$ \\
Hypercholesterolemia & 2.67 & $1.5-4.5$ & $<0.001$ \\
Smoking & 1.84 & $1.1-3.2$ & 0.028 \\
IVS1-397T $>C^{*} C$ allele & 2.99 & $1.3-6.5$ & 0.007 \\
\hline
\end{tabular}

$\mathrm{CAD}-=$ minimal coronary artery disease $; \mathrm{CAD}+=$ coronary artery disease $. \mathrm{OR}=$ odds ratio.

Table 4. Haplotype frequencies of $+261 G>C, I V S 1-397 T>C$ and $I V S 1-351 A>G$ polymorphisms in ESR1.

\begin{tabular}{|c|c|c|c|c|c|c|}
\hline \multirow[t]{2}{*}{ Haplotypes } & \multicolumn{2}{|c|}{$\begin{array}{c}\text { Control } \\
(\mathrm{N}=142)\end{array}$} & \multicolumn{2}{|c|}{$\begin{array}{c}\text { CAD- } \\
(N=111)\end{array}$} & \multicolumn{2}{|c|}{$\begin{array}{c}\text { CAD+ } \\
(N=192)\end{array}$} \\
\hline & $\mathrm{N}$ & $F(\%)$ & $\mathrm{N}$ & $F(\%)$ & $\mathrm{N}$ & $F(\%)$ \\
\hline G-C-G & 83 & 29.2 & 72 & 32.1 & 129 & 31.2 \\
\hline G-C-A & 12 & 4.2 & 4 & 1.8 & 14 & 3.4 \\
\hline G-T-G & 0 & 0.0 & 0 & 0.0 & 2 & 0.5 \\
\hline G-T-A* & 179 & 63.0 & 139 & 62.0 & 235 & $56.8^{a}$ \\
\hline C-C-A & 10 & 3.5 & 7 & 3.1 & 32 & 7.7 \\
\hline C-T-G & 0 & 0.0 & 0 & 0.0 & 1 & 0.2 \\
\hline C-T-A & 0 & 0.0 & 0 & 0.0 & 1 & 0.2 \\
\hline
\end{tabular}

$\mathrm{N}=$ number; $\mathrm{F}=$ frequency. $\mathrm{CAD}-=$ minimal coronary artery disease $; \mathrm{CAD}+=$ coronary artery disease.

${ }^{*} \mathrm{P}=0.039$, carriers of the most frequent haplotype (G-T-A) were compared with all

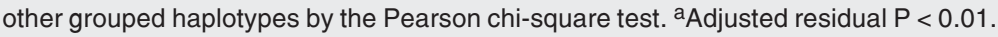


tion to ESR1 polymorphism and myocardial infarction. Shearman et al. (19) demonstrated that males in the Framingham Heart Study, carriers of the common ESR1 IVS1-397T >C $\mathrm{C} / \mathrm{C}$ genotype, have a substantial increase in risk of myocardial infarction and major atherosclerosis, while Schuit et al. (20) detected in postmenopausal women of the Rotterdam Study that carriers of the ESR1 haplotype 1 (IVS1-397T>C $* T$ allele and IVS1-351A> $G^{*} A$ allele) have an increased risk of myocardial infarction and ischemic heart disease whereas an absence of this association in men was reported in that study. As pointed out by Newton-Cheh and O'Donnell (36), there are some differences between the two studies, but a review of the available criteria points to no firm conclusion about which of the two studies should be regarded as the most valid. Several other studies also showed conflicting results. A beneficial effect of the IVS1-397T $>C * C$ allele has been reported in some studies. The presence of the IVSI$397 T>C^{*} C$ allele was protective against the risk of in-stent restenosis (18); postmenopausal women on hormone replacement therapy, homozygous for the $\mathrm{C}$ allele at the IVS1-397T>C site, showed less progression in atherosclerosis severity scores (22). However, negative effects of the same allele have also been described by others. Nordström et al. (17) showed that the IVSI-397T>C*C allele was associated with risk of aortic valve sclerosis and coronary atherosclerosis at angiography in women. Evidence that the IVSI$397 T>C$ site may modify coronary reactivity and LDL oxidation in healthy young men has also been reported (21). In that investigation adenosine-stimulated blood flow values were lower in subjects with the $\mathrm{C} / \mathrm{C}$ genotype at the IVS1-397T $>C$ site than in subjects with other genotypes, and plasma levels of oxidized LDL were on average higher in $\mathrm{C} / \mathrm{C}$ homozygous subjects than in subjects with $\mathrm{C} / \mathrm{T}$ and $\mathrm{T} / \mathrm{T}$ genotypes (21). Lehtimäki et al. (15) reported that the IVSI$397 T>C^{*} C$ allele was a risk factor for men aged 53 years or over, who have on average a larger area of complicated lesions.

The data for the Brazilian sample investigated here are in agreement with these findings. The IVS1-397T $>C^{*} C$ allele was associated with CAD severity, but this effect was not gender specific, because IVS1$397 T>C$ by gender interaction was not a significant variable in the logistic regression model. A $6 \%$ frequency of reduction of the $+261 G>C^{*} G-I V S 1397 T>C^{*} T-I V S 1$ $351 A>G^{*} A$ haplotype was detected in CAD+ subjects when compared to the CAD- and control samples (Table 4); this observation probably reflects the increased frequency of the IVS1-397T $>C * C$ allele in CAD+ subjects. These results are consistent with those reported by Shearman et al. (19) for males in the Framingham Heart Study.

In the present study, an effect of the $+261 G>C$ was also detected in the CAD sample, but restricted to males only. We have no explanation for this finding, but the differences in hormone dynamics between men and postmenopausal women are well known, and could account for the differences in association studies (20). The functional implications of the IVSI-397T $>C$, IVSI-35IA $>G$ and $+261 G>C$ polymorphisms have not been fully elucidated, but it has been speculated that genetic variants of ESR1 modify the expression or affinity of this receptor by estrogen. The $+261 G>C$ polymorphism is a silent mutation, the wild sequence $(+261 G>C * G$ allele) codes least frequently for alanine in humans $(7.1 \%$ of cases) and the silently mutated codon $\left(+261 G>C^{*} C\right.$ allele) codes most frequently (29.5\% of cases) (11). This might cause a modification of expression, but this hypothesis needs confirmation by expression studies. Herrington et al. (14) showed that IVSI$397 T>C * T$ allele eliminates a functional binding site for the transcription factor Bmyb, which suggests that the presence of this allele may result in lower ESR1 transcription. Moreover, these polymorphisms 
might only be co-segregating in linkage disequilibrium with a truly functional but so far unknown sequence variation elsewhere in the ESR1 gene. The long alleles of the TA repeat polymorphism in the promoter of the ESR1 gene have been associated with impaired coronary function and are in linkage disequilibrium with the variants investigated here, but other functional SNPs cannot be ruled out $(23,24)$.

In addition to the influence of estrogen on several factors involved in cardiovascular disease, such as lipid metabolism, coagulation and/or fibrinolysis, this hormone may be modifying the expression of various genes. Therefore, different polymorphisms may possibly exhibit association with different cardiovascular markers and also in opposite directions. According to Newton-Cheh and O’Donnell (36), "For both viewers and participants, the race for discoveries from genomic medicine will likely be a marathon, not a sprint", but the better identification of the relation between genomic variation and cardiovascular function may influence clinical decisions such as the use of hormone therapy for postmenopausal women and additionally will be helpful to identify the genetic susceptibility determinants of cardiovascular disease development.

\section{Acknowledgments}

Thanks are due to Ana Lúcia S. Antunes and Maria Perpétua de O. Pinto from the Clinical Analysis Laboratory of the Pharmacy College. We are also grateful to Dr. Marcos R. Torres, Dr. Alcides J. Zago, and to our colleagues Domingos L. Rios, André Vargas, Fabiana M. de Andrade, Fabiano Roldão Silveira, Marilu Fiegenbaum, and Marcel Arsand for help with sample collection, and to Dr. Sidia M. Callegari-Jacques for her advice about the statistical analysis.

\section{References}

1. Godsland IF (2001). Effects of postmenopausal hormone replacement therapy on lipid, lipoprotein, and apolipoprotein (a) concentrations: analysis of studies published from 1974-2000. Fertility and Sterility, 75: 898-915.

2. Mendelsohn ME \& Karas RH (1999). The protective effects of estrogen on the cardiovascular system. New England Journal of Medicine, 340: 1801-1811.

3. Hopkins PN \& Brinton EA (2003). Estrogen receptor 1 variants and coronary artery disease: shedding light into a murky pool. Journal of the American Medical Association, 290: 2317-2319.

4. Maas AH, van der Schouw YT, Grobbee DE et al. (2004). Rise and fall of hormone therapy in postmenopausal women with cardiovascular disease. Menopause, 11: 228-235.

5. Ohlsson C, Hellberg N, Parini P et al. (2000). Obesity and disturbed lipoprotein profile in estrogen receptor- $\alpha$-deficient male mice. Biochemical and Biophysical Research Communications, 278: 640645.

6. Hodgin JB, Krege JH, Reddick RL et al. (2001). Estrogen receptor $\alpha$ is a major mediator of 17ß-estradiol's atheroprotective effects on lesion size in apoe $/$ mice. Journal of Clinical Investigation, 107: 333-340.

7. Chen Z, Yuhanna IS, Galcheva-Gargova Z et al. (1999). Estrogen receptor $\alpha$ mediates the nongenomic activation of endothelial nitric oxide synthase by estrogen. Journal of Clinical Investigation, 103: 401-406.

8. Weel AEA, Uitterlinden AG, Westendorp ICD et al. (1999). Estrogen receptor polymorphism predicts the onset of natural and surgical menopause. Journal of Clinical Endocrinology and Metabolism, 84: 3146-3150.

9. Lorentzon M, Lorentzon R, Backstrom T et al. (1999). Estrogen receptor gene polymorphism, but not estradiol levels, is related to bone density in healthy adolescent boys: a cross-sectional and longitudinal study. Journal of Clinical Endocrinology and Metabolism, 84: 4597-4601.

10. Kobayashi S, Inoue S, Hosoi T et al. (1996). Association of bone mineral density with polymorphism of the estrogen receptor gene. Journal of Bone and Mineral Research, 11: 306-311.

11. Lehrer S, Rabin J, Kalir T et al. (1993). Estrogen receptor variant and hypertension in women. Hypertension, 21: 439-441.

12. Deng H-W, Li J, Li J-L et al. (2000). Association of estrogen receptor- $\alpha$ genotypes with body mass index in normal healthy postmenopausal Caucasian women. Journal of Clinical Endocrinology and Metabolism, 85: 2748-2751.

13. Herrington DM, Howard TD, Hawkins GA et al. (2002). Estrogenreceptor polymorphisms and effects of estrogen replacement on high-density lipoprotein cholesterol in women with coronary disease. Journal of Clinical Endocrinology and Metabolism, 346: 967974.

14. Herrington DM, Howard TD, Brosnihan KB et al. (2002). Common estrogen receptor polymorphism augments effects of hormone replacement therapy on E-selectin but not C-reactive protein. Circulation, 105: 1879-1882. 
15. Lehtimäki T, Kunnas TA, Mattila KM et al. (2002). Coronary artery wall atherosclerosis in relation to the estrogen receptor 1 gene polymorphism: an autopsy study. Journal of Molecular Medicine, 80: 176-180.

16. Lu H, Higashikata $\mathrm{T}$, Inazu A et al. (2002). Association of estrogen receptor- $\alpha$ gene polymorphisms with coronary artery disease in patients with familial hypercholesterolemia. Arteriosclerosis, Thrombosis, and Vascular Biology, 22: 1-7.

17. Nordström P, Glader CA, Dahlen G et al. (2003). Oestrogen receptor alpha gene polymorphism is related to aortic valve sclerosis in postmenopausal women. Journal of Internal Medicine, 254: 140146.

18. Ferrero V, Ribichini F, Matullo G et al. (2003). Estrogen receptoralpha polymorphisms and angiographic outcome after coronary artery stenting. Arteriosclerosis, Thrombosis, and Vascular Biology, 23: 2223-2228.

19. Shearman AM, Cupples LA, Demissie S et al. (2003). Association between estrogen receptor alpha gene variation and cardiovascular disease. Journal of the American Medical Association, 290: 22632270.

20. Schuit SC, Oei HH, Witteman JC et al. (2004). Estrogen receptor alpha gene polymorphisms and risk of myocardial infarction. Journal of the American Medical Association, 291: 2969-2977.

21. Lehtimäki T, Laaksonen R, Mattila KM et al. (2002). Oestrogen receptor gene variation is a determinant of coronary reactivity in healthy young men. European Journal of Clinical Investigation, 32 : 400-404.

22. Koivu TA, Fan Y-M, Mattila KM et al. (2003). The effect of hormone replacement therapy on atherosclerotic severity in relation to ESR1 genotype in postmenopausal women. Maturitas, 44: 29-38.

23. Kunnas TA, Laippala P, Penttilä A et al. (2000). Association of polymorphism of human $\alpha$ oestrogen receptor gene with coronary artery disease in men: a necropsy study. British Medical Journal, 321: $273-274$

24. Kunnas TA, Lehtimäki T, Karhunen PJ et al. (2004). Estrogen receptor genotype modulates myocardial perfusion in young men. Journal of Molecular Medicine, 82: 821-825.

25. Rios DL, Vargas AF, Ewald GM et al. (2003). Common variants in the lipoprotein lipase gene in Brazil: association with lipids and angiographically assessed coronary atherosclerosis. Clinical Chemistry and Laboratory Medicine: CCLM/FESCC, 41: 1351-1356.

26. Lahiri DK \& Nurnberger Jr JI (1991). A rapid non-enzymatic method for the preparation of HMW DNA from blood for RFLP studies. Nucleic Acids Research, 19: 5444.

27. Yaich L, Dupont WD \& Cavener DR (1992). Analysis of the Pvull restriction fragment-length polymorphism and exon structure of the estrogen receptor gene in breast cancer and peripheral blood. Cancer Research, 52: 77-83.

28. Andersen TI, Heimdal KR, Skrede M et al. (1994). Oestrogen receptor (ESR) polymorphisms and breast cancer susceptibility. Human Genetics, 94: 665-670.

29. Friedewald NT, Levy RI \& Fredrickson DS (1972). Estimation of the concentration of low-density lipoprotein cholesterol in plasma, without use of the preparative ultracentrifuge. Clinical Chemistry, 18: 499-502.

30. Abramson JH (2004). WINPEPI (PEPI-for-Windows): computer programs for epidemiologists. Epidemiologic Perspectives and Innovations, 1: 6.

31. Long JC, Williams RC \& Urbanek M (1995). An E-M algorithm and testing strategy for multiple-locus haplotypes. American Journal of Human Genetics, 56: 799-810.

32. Long JC (1999). Multiple Locus Haplotype Analysis, Version 2.0. Software and documentation distributed by the author. Section on population genetics and linkage. Laboratory of Neurogenetics, NIAAA, National Institutes of Health, Bethesda, MD, USA.

33. Peterson RJ, Goldman D \& Long JC (1999). Effects of worldwide population subdivision on ALDH2 linkage disequilibrium. Genome Research, 9: 844-852.

34. Schneider S, Kueffer J-M, Roessli D et al. (2000). Arlequin Version 2.000: A Software for Population Data Analysis. Genetics and Biometry Laboratory, University of Geneva, Geneva, Italy.

35. Lewontin RC (1988). On measures of gametic disequilibrium. Genetics, 120: 849-852.

36. Newton-Cheh C \& O'Donnell CJ (2004). Sex differences and genetic associations with myocardial infarction. Journal of the American Medical Association, 291: 3008-3010. 